\title{
Construcción y validación inicial de un cuestionario para evaluar la Calidad de Vida en mayores institucionalizados
}

\author{
$\mathbf{M}^{\mathrm{a}}$ del Mar Molero, $\mathbf{M}^{\mathrm{a}}$ del Carmen Pérez-Fuentes, \\ José J. Gázquez e Isabel Mercader \\ Universidad de Almería (España)
}

\begin{abstract}
El presente estudio tiene como objetivo la construcción y validación inicial de un instrumento específico para evaluar la Calidad de Vida de los mayores en contexto residencial. En primer lugar, se diseñó un cuestionario inicial Cuestionario de Evaluación de Calidad de Vida en Contexto Residencial (CECAVIR) que fue sometido a un proceso de depuración donde se reduce el número de ítems de 88 a 56. Posteriormente, se procedió a su implementación en una muestra de 50 sujetos mayores de 70 años institucionalizados y los resultados obtenidos en la nueva versión del mismo mostraron una mejora considerable en el coeficiente alfa, tanto en el cuestionario completo (de ,816 a ,865), como para cada una de sus escalas. Por otro lado, los resultados mostraron que la Capacidad Funcional es la dimensión que en mayor medida (44\%) explica la Calidad de Vida en mayores institucionalizados. Así, el CECAVIR se presenta como un instrumento específico de evaluación de la Calidad de Vida, que atiende a la multidimensionalidad de la misma y que obtiene óptimos resultados en los análisis iniciales de fiabilidad y validez, en población mayor institucionalizada.
\end{abstract}

Palabras clave: Calidad de Vida, cuestionario, validación, mayores institucionalizados.

Construction and initial validation of a questionnaire to assess Quality of Life in older institutionalized people. The aim of present study is the construction and initial validation of a specific instrument to assess Quality of Life of older people in a residential setting. Firstly, an initial questionnaire was designed, the Cuestionario de Evaluación de Calidad de Vida en Contexto Residencial (CECAVIR; in English, Questionnaire of Assessment of Quality of Life in Residential Setting) and subsequently refined, reducing the number of items from 88 to 56 . It was then administered to a sample of 50 institutionalized people over 70 years of age. The results obtained in the new version of the instrument showed a considerable improvement of the alpha coefficient, both in the total questionnaire (from .816 to .865), and in each subscale. The results also showed that Functional Capacity is the dimension that best explains (44\%) quality of life in older institutionalized people. Thus, the CECAVIR is presented as a specific instrument for the assessment of Quality of Life, which takes into account its multidimensionality, obtaining optimal results in the initial analyses of reliability and validity, in older institutionalized population.

Key words: Quality of Life, questionnaire, validation, older institutionalized people.

Correspondencia: María del Carmen Pérez Fuentes. Departamento de Psicología. Universidad de Almería. Ctra. de Sacramento, s/n. 04120. Almería (España). E-mail: $\underline{\text { mpf421@ @ual.es }}$ 
La tendencia demográfica actual se caracteriza por un envejecimiento progresivo de la población, en continuo crecimiento y de forma generalizada a nivel mundial (Naciones Unidas, 2011). Cada vez es mayor el número de personas que alcanzan edades avanzadas, aumentando también su representatividad con respecto a la población total. Esto conduce a la necesidad de reformular el objetivo de atención que hasta el momento era considerado con la llegada de la última etapa del ciclo vital. Así, no se trata de intervenir para aumentar el número de años, sino la calidad de los mismos o, lo que es lo mismo, de “dar vida a los años” (Bayarre, Pérez y Menéndez, 2008). Será a partir de esta reformulación de intenciones en la labor atencional, ya no meramente asistencial, cuando el concepto de Calidad de Vida se convierte en interés prioritario para aquellas disciplinas que trabajan para la mejora del bienestar de las personas mayores (Bayarre, 2009; Giró, 2006; IMSERSO, 2011; Zunzunegui y Béland, 2010).

La Calidad de Vida es un constructo cuya complejidad reside, por un lado, en su naturaleza multidimensional (Bayarre et al., 2008) y, por otro, en la importancia que tiene el contexto para su valoración (Fernández-Ballesteros, 1998). Así, cuando nos enfrentamos a la tarea de evaluar la Calidad de Vida, nos encontramos con una serie de dificultades basadas fundamentalmente en los problemas para su conceptualización. Debido a su naturaleza multidimensional, la Calidad de Vida se presenta como un constructo difícil de operacionalizar. De hecho, ha sido frecuentemente confundido con otros términos como bienestar (Díaz, Blanco y Durán, 2011), satisfacción (Davydov, Stewart, Richie y Chaudie, 2010) o felicidad (Browne et al., 1994). Otra de las controversias en la evaluación de la Calidad de Vida se refiere a la necesidad de atender no sólo a los componentes objetivos sino además a otro tipo de componentes de naturaleza subjetiva (Arita, Romano, García y Félix, 2005), con la dificultad que dicha inclusión entraña para su cuantificación (Cobb, 2000; Hernández, Rodríguez y Luciano, 2011). Por otro lado, se requiere una clara diferenciación de las características que definen la Calidad de Vida en un contexto determinado. Todo ello, con el fin de establecer una serie de indicadores de Calidad de Vida que resulten válidos ante unas circunstancias particulares, que se dan con frecuencia en el colectivo de mayores, como son las situaciones de institucionalización (Molina, Meléndez y Navarro, 2008).

Actualmente, existen numerosos estudios que evalúan la Calidad de Vida en personas con demencia (Duyckaerts y Litvan, 2008; Lucas-Carrasco, Peró y March, 2011), insuficiencia renal (Segui, Amador y Ramos, 2010), asma (Tan et al., 2009), diabetes mellitus (Hartman, 2008) o hipertensión (Hajjar et al., 2009). Concretamente, para este tipo de estudios en los que se valora la Calidad de Vida en una población que comparte el diagnóstico de una enfermedad determinada, los investigadores disponen de una amplia gama de instrumentos de Calidad de Vida específicos para cada patología (Mapi Research Trust, 2012). Por el contrario, cuando el punto en común de la muestra 
no es un diagnóstico, sino unas características determinadas o un contexto de referencia, la valoración de la Calidad de Vida se remite al uso de instrumentos genéricos como el World Health Organization Quality of Life Assessment Instrument (WHOQOL Group, 1995), que en su mayoría son utilizados indistintamente con población general. Otros instrumentos, como el SF-36 Health Survey (McHorney, Ware, Lu y Sherbourne, 1994) o el Sickness Impact Profile (Bergner, Bobbit, Pollard, Martin y Gilson, 1976), aún siendo ampliamente utilizados para medir la Calidad de Vida, fueron desarrollados inicialmente para la evaluación de la salud. Así, resulta inevitable pasar por alto el reducido número de instrumentos disponibles para evaluar, de manera específica, la Calidad de Vida en personas mayores (Gómez, Verdugo, Arias y Navas, 2008). Tradicionalmente, la medida de la Calidad de Vida ha estado en consonancia con las tendencias clásicas que, limitando su valoración a las dimensiones objetivas del constructo, se centraban casi de manera exclusiva en la dimensión salud (FernándezMayoralas, Fernández y Rojo-Pérez, 2005).

En torno al concepto de Calidad de Vida existen diversos componentes sobre los que se puede intervenir para su mejora: relaciones sociales y familiares, actividad y ocio, calidad ambiental o satisfacción con la vida, entre otros (Low y Molzhn, 2007). Uno de los componentes de la Calidad de Vida considerado de gran relevancia por la población mayor es la salud (Rodríguez-Artalejo, 2011), siendo los problemas que afectan a ésta los que más preocupan a este colectivo, sobre todo entre el género femenino (Medina y Carbonell, 2004). En el contexto institucional, aunque la valoración del estado de salud es baja, no se dan diferencias entre hombres y mujeres (Herrera et al., 2004). Otra dimensión de la Calidad de Vida en personas mayores, estrechamente relacionada con la salud, es la capacidad funcional (Gázquez, Pérez-Fuentes, Mercader y Molero, 2011; Jackson, 2006; Lobo, Santos y Carvarlho, 2007; Puga, 2002). La pérdida en el nivel de autonomía funcional puede dar lugar a situaciones de dependencia para la realización de AVD (Lusardi, Pellecchia y Shulman, 2003) y, en definitiva, graves alteraciones en la percepción de la capacidad de uno mismo, afectando negativamente en su Calidad de Vida (Albert et al., 2006). Al igual que ocurre con la salud, los datos obtenidos en la investigación apuntan hacia una mayor frecuencia de limitaciones de tipo funcional en las mujeres (Medina y Carbonell, 2004; Rojo, 2006). Por otro lado, con la llegada de la vejez la probabilidad de experimentar pérdidas sociales va en aumento, lo que se traduce en una grave alteración de las redes sociales con las que cuenta el individuo y, en definitiva, un deterioro de su Calidad de Vida (Winningham y Pike, 2007). En el caso de mayores institucionalizados, son los hombres los que en mayor medida sufren las consecuencias de la desadaptación social (Sáez, Meléndez y Aleixandre, 1995). Precisamente en el entorno residencial, el ocio se presenta como una herramienta clave para la creación y mejora de redes sociales y, por tanto, una de las dimensiones a tener en cuenta para la mejora de la Calidad de Vida de los residentes 
(Hernández, 2009). Otro aspecto de la Calidad de Vida en mayores, a menudo subestimado, es la calidad ambiental o las características y disposición del ambiente en el que la persona mayor se desenvuelve. Concretamente, se hace referencia a la función que el entorno físico tiene para facilitar o no, el desarrollo autónomo de la persona que reside en él (Chaudhury, 2003; Hwang y Ziebarth, 2006). Las características del ambiente están directamente relacionadas con el grado de satisfacción de los residentes y, por tanto, con el deseo de permanencia y adaptación a ese nuevo ambiente que es la residencia (Cerina y Fornara, 2011); por lo que, la adecuación de las características físicas en el entorno residencial, puede llegar a paliar el declive en otras dimensiones de la Calidad de Vida (Vargas y Gallego, 2005). Por último, existe otro aspecto integrado en el estudio de la Calidad de Vida en la población mayor: la satisfacción con la vida, la cual implica un juicio por parte del sujeto acerca de su trayectoria vital. Hay estudios que se han dedicado a analizar la relación entre la satisfacción vital y la edad, observándose en algunos casos una ligera disminución en la satisfacción vital conforme el individuo avanza en años (Navarro, Meléndez y Tomás, 2008; Triadó, 2011). No obstante, al ser esta relación muy baja, se sigue apoyando la idea generalizada de que la satisfacción vital es una variable que se mantiene bastante estable con la edad y especialmente en población de más de 65 años (Meléndez, Navarro, Oliver y Tomas, 2009).

En las últimas décadas, se pone de manifiesto la importancia de atender al carácter multidimensional y global de la Calidad de Vida, con una evaluación orientada a la mejora cualitativa, no sólo de la salud, sino también del resto de sus componentes (Bayarre, Pérez y Menéndez, 2008). Por todo ello, se hace cada vez más necesario el desarrollo, adaptación y validación de instrumentos para la valoración de la Calidad de Vida dirigidos a la población mayor, y no sólo atendiendo a la presencia o no de enfermedad, como es el caso de los instrumentos específicos actuales. En la medida en que se adecue el instrumento de evaluación a las circunstancias particulares del individuo, con especial atención al contexto, nos aproximaremos a una evaluación más válida y fiable de la Calidad de Vida. Así, el objetivo de nuestra investigación es el desarrollo de un instrumento específico para evaluar la Calidad de Vida en población mayor institucionalizada.

\section{MÉTODO}

\section{Fase I. Diseño y elaboración del cuestionario}

En primer lugar, se llevó a cabo una revisión bibliográfica para conocer las variables relacionadas con la Calidad de Vida, así como los diferentes aspectos relacionados con su valoración y las características propias de la población mayor institucionalizada. Esta revisión teórica permitió formular un total de 88 ítems, que conforman la versión inicial del Cuestionario de Evaluación de Calidad de Vida en Contexto Residencial (CECAVIR). Todos los ítems, de respuesta cerrada con cinco 
alternativas de respuesta, se distribuyen en siete hojas de aplicación: la primera destinada a la recogida de datos personales y las seis restantes se corresponden con cada una de las dimensiones estimadas para la valoración de la Calidad de Vida en población mayor institucionalizada (Salud, Relaciones Sociales y Familia, Actividad y Ocio, Calidad Ambiental, Capacidad Funcional y Satisfacción con la Vida).

Posteriormente, con objeto de evaluar el nivel de comprensión y así proceder a la redacción definitiva de los ítems, se aplicó esta primera versión del CECAVIR a una muestra piloto de 30 sujetos, de características similares a la muestra que posteriormente será utilizada para la implementación del cuestionario. Además, para su revisión se tuvo en cuenta la frecuencia en las respuestas de la muestra piloto, siendo eliminadas aquellas cuestiones con bajo poder de discriminación.

Para la depuración del cuestionario en su propuesta inicial, cada una de las escalas de Calidad de Vida, se analizó de forma independiente. Se llevó a cabo el análisis de fiabilidad de las escalas, mediante el cálculo del coeficiente alfa de Cronbach. A partir de los resultados obtenidos, se identificaron aquellos ítems que presentaban baja consistencia interna y por tanto debían ser eliminados del cuestionario.

La versión final del CECAVIR estuvo formada por un total de 56 ítems, de los cuáles 7 estaban destinados a la recogida de datos personales y los 48 restantes a evaluar las diferentes dimensiones de Calidad de Vida propuestas inicialmente.

\section{Fase II. Implementación del cuestionario}

Para la implementación del cuestionario, se seleccionaron 50 sujetos mayores de 70 años, todos ellos usuarios de una residencia de ancianos de carácter público. Con una media de edad de 81,26 años $(D T=5,889)$, siendo la edad mínima de los participantes de 72 años y la máxima de 94 años. Del total de la muestra, el 54\% eran hombres y el $46 \%$ mujeres, con una media de edad de 80,78 (DT=5,866) y 81,83 (DT= 5,997) años, respectivamente. La modalidad de aplicación fue individual, mediante entrevista estructurada, y con un tiempo estimado para su cumplimentación de entre 2025 minutos por sujeto.

Tras su aplicación, se determinó la consistencia interna del cuestionario mediante el coeficiente alfa de Cronbach y, para comprobar la validez de constructo, se llevó a cabo un análisis factorial exploratorio con el Método de Componentes Principales. Además, se aplicó la correlación de Pearson y se llevó a cabo un análisis de Regresión Múltiple paso a paso para conocer la influencia y el valor de cada uno de los factores implicados en la valoración de la Calidad de Vida. Por último, para los análisis de puntuaciones medias y conocer si existen diferencias significativas entre los grupos de género, se aplicó la prueba $t$ de Student para muestras independientes. 


\section{RESULTADOS}

\section{Depuración de ítems y consistencia interna}

La tabla 1 contiene la media, varianza y el coeficiente alfa para el cuestionario definitivo, que en su versión inicial contaba con seis escalas (Salud, Relaciones Sociales y Familia, Actividad y Ocio, Calidad Ambiental, Capacidad Funcional y Satisfacción con la Vida) y un total de 88 ítems (incluidos los 7 ítems para la recogida de datos personales). En el cuestionario inicial, recordamos que el coeficiente alfa era de ,816 y, tras la depuración de los ítems en cada una de las escalas que lo integran, a pesar de de ser más breve (con un total de 56 ítems, de los cuáles 48 ítems se distribuyen en 5 escalas), el coeficiente alfa en el cuestionario final alcanza un valor de ,865.

Tabla 1. Estadísticos descriptivos y coeficiente alfa de Cronbach para el cuestionario final

\begin{tabular}{ccccc}
\hline $\mathrm{N}^{\circ}$ de ítems & Media & Varianza & Desviación Típica & Alpha de Cronbach \\
\hline 48 & 153,62 & 542,159 & 23,284 &, 865 \\
\hline
\end{tabular}

Por otro lado, a partir del análisis de fiabilidad para cada una de las escalas que integran el cuestionario, se obtienen los siguientes resultados (Tabla 2):

Tabla 2. Valores de alfa de Cronbach, por escala y total del cuestionario en su versión inicial y tras la depuración

\begin{tabular}{|c|c|c|c|c|c|}
\hline & & \multicolumn{2}{|c|}{ Cuestionario Inicial } & \multicolumn{2}{|c|}{ Cuestionario Final } \\
\hline & & Ítems & $\alpha$ & Ítems & $\alpha$ \\
\hline & Cuestionario Completo & 81 &, 816 & 48 & ,865 \\
\hline \multirow{5}{*}{ 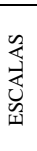 } & Salud & 17 &, 035 & 9 &, 548 \\
\hline & Relaciones Soc. y Fam. & 16 &, 648 & 13 & ,757 \\
\hline & Actividad y Ocio & 15 & 680 & 9 & 901 \\
\hline & Calidad Ambiental & 14 & ,886 & 12 & ,910 \\
\hline & Capacidad Funcional & 13 &, 564 & 5 & ,935 \\
\hline
\end{tabular}

En la escala Salud, de los 17 ítems de la versión inicial, éstos se reducen a nueve. El coeficiente alfa, en este caso, es el más bajo de todas las escalas que integran el cuestionario, con un valor de ,548. Aunque, por otro lado, tras la depuración de la escala, éste resulta ser el valor de alfa que ha sufrido una mejora más notable con respecto a su versión inicial $(\alpha=, 035)$. La escala Relaciones Sociales y Familia ve reducido su número de ítems tras la depuración, de 16 a 13. En este caso, con la eliminación de tan solo tres elementos, se consigue una mejora del coeficiente alfa, alcanzando éste un ,757 en el cuestionario final (frente a un ,648 en la versión inicial). Por otro lado, el número de elementos que integra la escala Actividad y Ocio también ve reducido su número tras el proceso de depuración, pasando de 15 a 9 ítems, y aumentando el valor del coeficiente alfa de ,680 a ,901 en el cuestionario final. La escala Calidad Ambiental, a pesar de ser la que mejor coeficiente de fiabilidad presentó en la versión inicial del cuestionario $(\alpha=, 886)$, con la eliminación de dos de sus elementos se obtiene un valor de alfa de ,91 para su versión final. Finalmente, una de las escalas que, 
tras la depuración, ha visto reducido el número de sus elementos en mayor medida es la escala Capacidad Funcional: de 13 ítems de la versión inicial a 5 ítems en su versión final. A pesar de la importante reducción de ítems, se obtiene para esta escala el coeficiente alfa más elevado: ,935, frente a un valor alfa de ,564 previo al proceso de depuración. En cuanto a la escala de Satisfacción con la Vida, tras la depuración, ésta queda eliminada como tal, debido a la existencia de una covarianza promedio negativa entre sus elementos. En este caso, optamos por formular un único ítem: "Actualmente, en general me va bien, me siento bien".

En resumen, los datos extraídos a partir del análisis de fiabilidad, muestran que tanto para el cuestionario completo como para cada una de sus escalas, se produce una mejora considerable en el coeficiente alfa tras la depuración, llegando en algunos casos a situarse éste muy próximo a 1, como ocurre con la escala Capacidad Funcional (Tabla 2).

\section{Análisis factorial exploratorio}

En la tabla 3, se recogen los resultados obtenidos a partir del análisis factorial de Componentes Principales para cada una de las escalas del cuestionario.

Tabla 3. Estructura factorial y varianza explicada (\%) para las escalas del cuestionario

\begin{tabular}{|c|c|c|c|c|c|}
\hline Escala Salud & Factor 1 & & Factor 2 & Factor 3 & Factor 4 \\
\hline Varianza explicada (\%) & 29,409 & & 22,159 & 15,129 & 11,615 \\
\hline Varianza total $(\%)$ & \multicolumn{5}{|c|}{78,311} \\
\hline Escala Relaciones Sociales y Familia & Factor 1 & Factor 2 & Factor 3 & Factor 4 & Factor 5 \\
\hline Varianza explicada (\%) & 27,178 & 25,022 & 12,875 & 8,862 & 8,368 \\
\hline Varianza total $(\%)$ & & & 82,304 & & \\
\hline Escala Actividad y Ocio & \multicolumn{3}{|c|}{ Factor 1} & \multicolumn{2}{|c|}{ Factor 2} \\
\hline Varianza explicada (\%) & \multicolumn{3}{|c|}{60,032} & \multicolumn{2}{|c|}{12,760} \\
\hline Varianza total $(\%)$ & \multicolumn{5}{|c|}{72,793} \\
\hline Escala Calidad Ambiental & Factor 1 & & Factor 2 & \multicolumn{2}{|c|}{ Factor 3} \\
\hline Varianza explicada (\%) & 54,401 & & 13,442 & \multirow{2}{*}{\multicolumn{2}{|c|}{9,093}} \\
\hline Varianza total $(\%)$ & \multirow{2}{*}{\multicolumn{5}{|c|}{$\frac{76,935}{\text { Factor } 1}$}} \\
\hline Escala Capacidad Funcional & & & & & \\
\hline $\begin{array}{r}\text { Varianza explicada (\%) } \\
\text { Varianza total }(\%)\end{array}$ & \multicolumn{5}{|c|}{79,862} \\
\hline
\end{tabular}

Tal y como se observa en la tabla 3, se obtiene una estructura de cuatro factores para la escala Salud del cuestionario, que explican el 78,3\% de la varianza total para la escala. El Factor 1 explica un 29,4\% de la varianza total. Está constituido por los ítems 8, 10 y 11; relacionados con la calidad del sueño y el descanso. El Factor 2 supone un $22,1 \%$ de la varianza total, y está constituido por los ítems $9,13,14$ y 16; referentes a la valoración del estado de salud propio y el estado de salud de familiares cercanos. El Factor 3, explica un $15,1 \%$ de la varianza total e incluye el ítem 12, que hace referencia a la presencia o no de problemas relacionados con las comidas. Por último, el Factor 4 
explica un 11,6\% de la varianza total. Consta de un solo ítem (15), que valora la calidad de la asistencia sanitaria recibida.

Para la escala Relaciones Sociales y Familia, se extraen cinco factores que explican el $82,3 \%$ de la varianza total para la escala. El Factor 1 explica un 27,17\% de la varianza total. Está constituido por los ítems 17, 18, 19, 21, 22 y 25, relacionados con la frecuencia de los contactos familiares en residencia, así como el grado de satisfacción que el sujeto tiene con los mismos. El Factor 2 supone un $25 \%$ de la varianza total, y está constituido por los ítems 26, 27, 28 y 29, asociados a la valoración de las relaciones sociales en residencia. El Factor 3, explica un 12,8\% de la varianza total e incluye el ítem 20, que hace referencia al hecho de haber sido atendido por los hijos en algún momento anterior al ingreso en residencia. El Factor 4, supone un 8,8\% de la varianza total e incluye el ítem 23, relacionado con las visitas de los hermanos/as. Por último, el Factor 5 explicaría un $8,3 \%$ de la varianza total, consta de un solo ítem (24), que trata sobre el conocimiento de problemas familiares como fuente de preocupación para el sujeto en residencia.

La estructura factorial de la escala Actividad y Ocio consta de dos factores que explican el $72,7 \%$ de la varianza total para la escala. El Factor 1 explica el $60 \%$ de la varianza total. Está constituido por casi todos los ítems de la escala (30,32-38), excepto el ítem 31 que corresponde al Factor 2 y hace referencia al ocio relacionado con la socialización en residencia. Este último factor da cuenta de un 12,7\% de la varianza total.

Como se puede observar en la tabla 3, se obtiene una estructura de tres factores para la escala Calidad Ambiental. Entre estos tres factores se explica el 76,93\% de la varianza total para la escala. La varianza del Factor 1 supone un 54,40\% de la varianza total. Está constituido por los ítems 39, 41, 42, 43, 44, 45, 48, 49 y 50, que se refieren a la valoración y satisfacción del sujeto con el entorno físico de la residencia. El Factor 2, explica un 13,4\% de la varianza total e incluye el ítem 40 sobre la valoración sobre la temperatura en residencia. Por último, el Factor 3 explica un $9 \%$ de la varianza total, consta de los ítems 46 y 47, relacionados con la valoración de los servicios en cuanto a la cobertura de necesidades del propio residente.

Finalmente, para la escala Capacidad Funcional se extrae un solo factor, que coincide con el total de la varianza para la escala $(79,8 \%)$.

Análisis de correlaciones entre las escalas y diferencia de medias según género

Tal y como se observa en la tabla 4 , se da una correlación positiva y significativa entre las escalas Salud y Relaciones Sociales y Familia, lo que sugiere que a medida que la salud mejora, las relaciones sociales y familiares son valoradas más positivamente por los residentes. 
Tabla 4. Correlaciones de Pearson entre las puntuaciones totales de las escalas de Calidad de Vida en el

\begin{tabular}{lccccc}
\multicolumn{7}{c}{ CECAVIR } \\
\hline & Total S & Total RSF & Total AO & Total CA & Total CF \\
\hline Total S & 1 &, $485^{* *}$ &, 227 &, 097 &, 110 \\
Total RSF & 1 &, 053 &, 100 &, $321^{*}$ \\
Total AO & & & 1 &, $608^{* *}$ &,$- 307^{*}$ \\
Total CA & & & & 1 &, 048 \\
Total CF & & & & & 1 \\
\hline
\end{tabular}

Se observa también, una correlación positiva y significativa entre las escalas Calidad Ambiental y Actividad y Ocio, que indica que a mayor satisfacción con el entorno residencial, mayor será la participación y satisfacción con el ocio que allí se desarrolla. Por otro lado, la escala Capacidad Funcional presenta una correlación positiva significativa con Relaciones Sociales y Familia y una correlación negativa y significativa con Actividad y Ocio. Esto sugiere que cuanto mayor es la necesidad de ayuda para las actividades de la vida diaria, la valoración de las relaciones sociales y familiares es más positiva, pero la participación/satisfacción con el ocio es menor.

Para los totales de las escalas, en función del género no se dan diferencias significativas entre los grupos. Aunque se observa como los hombres tienden a obtener puntuaciones medias más elevadas para todas las escalas del cuestionario, excepto para la Capacidad funcional, siendo las mujeres las que obtienen aquí una media más elevada (Tabla 5).

Tabla 5. Totales escalas Calidad de Vida y género. Descriptivos y prueba $t$ para la igualdad de medias

\begin{tabular}{|c|c|c|c|c|c|c|c|c|}
\hline \multirow{3}{*}{ Escalas } & \multicolumn{6}{|c|}{ Género } & \multirow{3}{*}{$t$} & \multirow{3}{*}{ Sig. } \\
\hline & \multicolumn{3}{|c|}{ Hombres } & \multicolumn{3}{|c|}{ Mujeres } & & \\
\hline & $\mathrm{N}$ & Media & DT & $\mathrm{N}$ & Media & DT & & \\
\hline Salud & 27 & 3,38 & ,474 & 23 & 3,28 & ,641 & ,613 & ,543 \\
\hline Relaciones Sociales y Familia & 27 & 3,13 & ,507 & 23 & 2,90 &, 736 & 1,291 & ,203 \\
\hline Actividad y Ocio & 27 & 3,19 & 1,126 & 23 & 3,09 & 1,087 & ,336 & ,738 \\
\hline Calidad Ambiental & 27 & 4,01 &, 705 & 23 & 3,99 &, 609 &, 121 & ,905 \\
\hline Capacidad Funcional & 27 & 2,14 & 1,509 & 23 & 2,26 & 1,465 &,- 267 & ,791 \\
\hline
\end{tabular}

\section{Modelo de Regresión}

Con el objeto de determinar qué dimensiones o escalas explican la Calidad de Vida, y en qué medida lo hace cada una de ellas, se lleva a cabo un análisis de Regresión Múltiple (pasos sucesivos). Para ello, se considera como variable dependiente la Calidad de Vida y como variables independientes las puntuaciones totales para cada una de las dimensiones de Calidad de Vida incluidas en el cuestionario. El Modelo de Regresión resultante se muestra en la siguiente figura: 


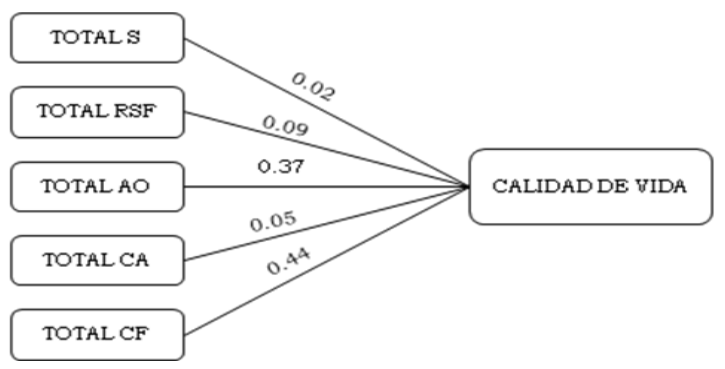

Nota: Total S (Puntuación Total de la Escala Salud); Total RSF (Puntuación Total de la Escala Relaciones Sociales y Familia); Total AO (Puntuación Total de la Escala Actividad y Ocio); Total CA (Puntuación Total de la Escala Calidad Ambiental); Total CF (Puntuación Total de la Escala Capacidad Funcional).

Así, tal y como se observa, la Calidad de Vida estaría explicada en un $44 \%$ por la dimensión de Capacidad Funcional $\left(R^{2}=, 449\right)$, un $37 \%$ por la Actividad y Ocio $\left(R^{2}=, 373\right)$, un $9 \%$ por las Relaciones Sociales y Familia $\left(R^{2}=, 097\right)$, un 5\% por la Calidad Ambiental $\left(R^{2}=, 051\right)$, y por último, tan sólo un $2 \%$ de la Calidad de Vida sería explicada por la dimensión Salud $\left(R^{2}=, 029\right)$.

\section{DISCUSIÓN}

La naturaleza multidimensional de la Calidad de Vida, junto a la dificultad que entraña su cuantificación (Cobb, 2000; Hernández et al., 2011), hacen de su evaluación una tarea costosa. El cuestionario desarrollado en el presente estudio, CECAVIR, pretende dar respuesta a la necesidad de contar con un instrumento específico que permita evaluar la Calidad de Vida de los mayores institucionalizados.

Tras el proceso de depuración del cuestionario, se obtienen resultados satisfactorios en tanto que se reduce considerablemente el número de ítems que lo componen, al mismo tiempo que se produce una mejora en la consistencia interna del cuestionario completo y también para cada una de sus escalas. Un coeficiente alfa de Cronbach de ,86 para la calificación total, mayor que la obtenida por algunas de las escalas por separado, confirma el carácter multidimensional del constructo de Calidad de Vida (Bayarre et al., 2008). Los valores alfa de Cronbach para cuatro de las cinco escalas del cuestionario resultaron superiores a ,60, llegando a un máximo de ,93 en la escala Capacidad Funcional. Por el contrario, la dimensión Salud obtuvo el valor más bajo $(\alpha=, 54)$, aunque se mantuvo como una escala del cuestionario por su relevancia entre la población mayor (Rodríguez-Artalejo, 2011) y con la intención de su revisión en futuras aplicaciones del cuestionario. Por otro lado, la estrecha relación que la salud mantiene con otras dimensiones como la Capacidad Funcional (Gázquez et al., 2011; Jackson, 2006; Lobo et al., 2007; Puga, 2002), nos lleva a plantear la posibilidad de 
integrar ambas escalas, de manera que se adopte un concepto de salud funcional más ajustado a las características de la población mayor institucionalizada.

Por otro lado, los resultados del análisis factorial exploratorio para las escalas del cuestionario revelan valores de la varianza total explicada que superan en todos los casos el 70\%. Los factores identificados en el análisis factorial coinciden conceptualmente con la propuesta teórica considerada inicialmente para cada dimensión de la Calidad de Vida. La existencia de correlaciones significativas entre las escalas confirma no solo la multidimensionalidad del constructo de Calidad de Vida, sino además la interrelación entre las diferentes dimensiones que lo componen (Hernández, 2009; Vargas y Gallego, 2005). En cuanto al género, a pesar de no darse diferencias significativas entre los grupos (Herrera et al., 2004), a partir del análisis de medias se observa que son las mujeres las que tienden a presentar puntuaciones medias superiores a los hombres en la escala Capacidad Funcional. Es necesario aclarar que esta es una escala que se mide en términos de necesidad de ayuda, por lo que puntuaciones más elevadas en esta indican un peor estado funcional (Medina y Carbonell, 2004; Rojo, 2006).

Finalmente, es de destacar el hecho de que la salud, siendo un aspecto tan valorado por la población mayor (Rodríguez-Artalejo, 2011; Medina y Carbonell, 2004) tan sólo explique un $2 \%$ de la Calidad de Vida en población mayor institucionalizada, tal y como muestra el modelo de regresión. Resultados como estos pueden estar explicados por la influencia del contexto (Fernández-Ballesteros, 1998) y la propia situación de institucionalización (Molina et al., 2008), que hace que todo el peso de la valoración que el sujeto hace de su estado de salud recaiga sobre la capacidad funcional.

El CECAVIR se presenta como un instrumento específico de evaluación de la Calidad de Vida que atiende a tres aspectos fundamentales: la multidimensionalidad de la Calidad de Vida, las características de la población mayor y la influencia del contexto residencial. Aunque el instrumento desarrollado presenta una fiabilidad y una validez aceptables, los resultados obtenidos han de ser tomados con cautela debido a la principal limitación del estudio que es el reducido tamaño de la muestra. Por lo que superar dicha limitación será objetivo prioritario en futuras aplicaciones del cuestionario.

\section{REFERENCIAS}

Albert, S., Lehman, J., Burkhardt, A., Merete-Roa, B., Noboa-Lemonier, R. y Teresi, J. (2006). Variation in Sources of Clinician-Rated and Self-Rated instrumental activities of daily living disability. Journal of Gerontology and Medicine Sciences, 61, 826-831.

Arita, B.Y., Romano, S., García, N. y Félix, M.R. (2005). Indicadores objetivos y subjetivos de la calidad de vida. Enseñanza e Investigación en Psicología, 10(1), 93-102.

Bayarre, H.D. (2009). Calidad de vida en la tercera edad y su abordaje desde una perspectiva cubana. Revista Cubana de Salud Pública, 35(4), 110-116. 
Bayarre, H.D., Pérez, J. y Menéndez, J. (2008). Las transiciones demográfica y epidemiológica y la calidad de vida objetiva en la tercera edad. Geroinfo, 1(3).

Bergner, M., Bobbit, R.A., Pollard, W.E., Martin, D.P. y Gilson, B.S. (1976). The Sickness impact Profile: validation of a health status measure. Medical Care, 14, 57-67.

Browne, J.P., O'Boyle, C.A., McGee, H.M., Joyce, C.R.B., McDonald, N.J., O'Malley, K. у Hiltbrunner, B. (1994). Individual quality of life in the healthy elderly. Quality of life Research, 3, 244-325.

Cerina, V. y Fornara, F. (2011). Los determinantes psicológicos de las actitudes hacia el traslado en las personas mayores: una encuesta en entornos urbanos y rurales. Bilingual Journal of Environmental Psychology, 2(3), 271-285.

Chaudhury, H. (2003). Quality of life and place-therapy. Journal Housing Elderly, 17(2), 85-103.

Cobb, C. (2000). Measurement Tools and Quality of Life. San Francisco: Redefining Progress.

Davydov, D., Stewart, R., Ritchie, K. y Chaudieu, I. (2010). Resilience and mental health. Clinical Psychology Review, 30, 479-495.

Díaz, D., Blanco, A. y Durán, M.M. (2011). The Structure of well-being: The empirical encounter of three traditions. Revista de Psicología Social, 26(3), 357-372.

Duyckaerts, C. y Litvan, I. (2008). Dementias: Handbook of clinical neurology. San Francisco: University of California.

Fernández-Ballesteros, R. (1998). Vejez con Éxito o vejez competente: un reto para todos. Ponencias de las IV Jornadas de la AMG: Envejecimiento y Prevención. Barcelona: AMG.

Fernández-Mayorales, G., Rojo, F., Abellán, A. y Rodríguez, V. (2003). Envejecimiento y salud. Diez años de investigación en el CSIC. Revista Multidisciplinar de Gerontología, 13(1), 43-46.

Gázquez, J.J., Pérez-Fuentes, M.C., Mercader, I. y Molero, M.M. (2011). Prevalencia de la dependencia funcional en personas mayores. Anales de Psicología, 27(3), 871-876.

Giró, J. (2006). El envejecimiento activo en la sociedad española. En J. Giró (Coord.), Envejecimiento activo, envejecimiento en positivo (pp. 15-36). La Rioja: Universidad de La Rioja.

Hajjar, I., Dickson, B., Blackledge, J., Lewis, P., Herman, J. y Watkins, K. (2009). A multidisciplinary management program in primary care to improve hypertension control and healthy behaviors in elderly patients. Journal of American Geriatry Society, 55(4), $1-4$.

Hartman, I. (2008). Insulin analogs: impact on treatment success, satisfaction, quality of life, and adherence. Clinical Medical Research, 6(2), 1-9.

Hernández, G. (2009). Cese de la actividad profesional y preparación para la jubilación. Cuadernos de Relaciones Laborales, 28(1), 63-81.

Hernández, M., Rodríguez, M. y Luciano, M.C. (2011). Contextual control and generalization of say-do correspondence. A preliminary study. International Journal of Psychology and Psychological Therapy, 11(2), 269-284.

Hwang, E. y Ziebarth, A. (2006). Impacts of residential environments on housing satisfaction among Korean-American elders. Housing and Society, 33(2), 1-20.

IMSERSO (2011). Envejecimiento Activo. Libro Blanco. Madrid: IMSERSO.

Jackson, A.S. (2006). The evolution and validity of health-related fitness. Queso, 58, 160-175.

Lobo, A., Santos, M.P. y Carvalho, J. (2007). Anciano institucionalizado: calidad de vida y funcionalidad. Revista Española de Geriatría y Gerontología, 42(1), 22-26.

Low, G. y Molzhn, A.E. (2007). Predictors of quality of life in old age: a cross validation study. Research in Nursing and Health, 30(2), 141-150. 
Lucas-Carrasco, R., Peró, M. y March, J. (2011). Calidad de vida global en personas con demencia. Revista de Neurología, 52, 139-146.

Lusardi, M., Pellecchia, G. y Schulman, M. (2003). Functional performance in community living older adults. Journal of Geriatric Physical Therapy, 26, 14-20.

Mapi Research Trust (2012). PROQOLID, Patient-Reported Outcome and Quality of Life Instruments Database. http://www.proqolid.org/

McHorney, C.A., Ware, J.E., Lu, R. y Sherbourne, C.D. (1994). The MOS 36-item short form health survey (SF-36): III. Tests of data quality, scaling assumptions, and reliability across diverse patient groups. Medical Care, 32, 40-66.

Medina, M.E. y Carbonell, M.C. (2004). Evaluación de la calidad de vida de las personas mayores en el municipio de Murcia. Portularia, 4, 179-186.

Meléndez, J.C., Navarro, E., Oliver, A. y Tomás, J.M. (2009). La satisfacción vital en los mayores. Boletín de Psicología, 95, 29-42.

Molina, C., Meléndez, J.C. y Navarro, E. (2008). Bienestar y calidad de vida en ancianos institucionalizados y no institucionalizados. Anales de Psicología, 24(2), 312-319.

Navarro, E., Meléndez, J.C. y Tomás, J.M. (2008). Análisis del bienestar en la vejez según la edad. Revista Española de Geriatría y Gerontología, 43, 90-95.

Puga, M.D. (2002). Dependencia y necesidades asistenciales de los mayores en España. Previsión del año 2010. Madrid: Fundación Pfizer.

Rodríguez-Artalejo, F. (2011). Envejecer satisfactoriamente. Revista Española de Geriatría y Gerontología, 46(1), 1-2.

Rojo, F. (2006). Población mayor, calidad de vida y familia: demanda y prestación de cuidados en el seno familiar. Madrid: IMSERSO.

Sáez, N., Meléndez, J.C. y Aleixandre, M. (1995). Introducción a la Gerontopsicología. Valencia: Promolibro.

Segui, A., Amador, P. y Ramos, A.B. (2010). Calidad de vida en pacientes con insuficiencia renal crónica en tratamiento con diálisis. Revista Sociedad Española de Enfermería y Nefrología, 13(3), 155-160.

Tan, H. et al. (2009). Impact of Asthma Controller Medications on Clinical, Economic, and Patient-Reported Outcomes. Mayo Clinical Proceedures, 84(8), 1-11.

Triadó, C. (2011). Personalidad y adaptación en la vejez. Informaciones Psiquiátricas, 204(3), 9599.

WHOQOL Group (1995). The World Health Organization Quality of life assessment (WHOQOL). Position Paper from the World Health Organization. Society of Science and Medicine, 4l(10), 1403-1409.

Winningham, R.G. y Pike, N.L. (2007). A cognitive intervention to enhance institutionalized older adults social support networks and decrease loneliness. Aging Mental Health, 11, 716721.

Zunzunegui, M.V. y Béland, F. (2010). Políticas intersectoriales para abordar el reto del envejecimiento activo. Informe SESPAS 2010. Gaceta Sanitaria, 24(1), 68-77.

Recibido: 6 de marzo de 2012

Recepción Modificaciones: 1 de abril de 2012

Aceptado: 3 de abril de 2012 